\title{
Probing the role of long-range interactions in the dynamics of a long-range Kitaev Chain
}

\author{
Anirban Dutta and Amit Dutta \\ Department of Physics, Indian Institute of Technology, Kanpur-208016, India
}

\begin{abstract}
We study the role of long-range interactions (more precisely, the long-range superconducting gap term) on the non-equilibrium dynamics considering a long-range $p$-wave superconducting chain in which superconducting term decays with distance between two sites in a power-law fashion characterised by an exponent $\alpha$. We show that the Kibble-Zurek scaling exponent, dictating the power-law decay of the defect density in the final state reached following a slow (in comparison to the time-scale associated with the minimum gap in the spectrum of the Hamiltonian) quenching of the chemical potential $(\mu)$ across a quantum critical point, depends non-trivially on the exponent $\alpha$ as long as $\alpha<2$; on the other hand, for $\alpha>2$, one finds that the exponent saturates to the corresponding well-know value of $1 / 2$ expected for the short-range model. Furthermore, studying the dynamical quantum phase transitions manifested in the non-analyticities in the rate function of the return possibility $(I(t))$ in subsequent temporal evolution following a sudden change in $\mu$, we show the existence of a new region; in this region, we find three instants of cusp singularities in $I(t)$ associated with a single sector of Fisher zeros. Notably, the width of this region shrinks as $\alpha$ increases and vanishes in the limit $\alpha \rightarrow 2$ indicating that this special region is an artefact of long-range nature of the Hamiltonian.
\end{abstract}

\section{INTRODUCTION}

Remarkable experimental advancement in closed quantum systems of ultracold atoms trapped in optical lattices [1, 2] and corresponding studies of non-equilibrium dynamics 3 8 of many-body systems have triggered in a plethora of theoretical studies. At the same time, numerous experimental studies of light-induced systems have resulted in the possibility of non-equilibrium superconductivity and emergent topological systems [9, 10]. In parallel, the theoretical studies investigate the growth of entanglement entropy following a quench [11, thermalization 12, light-induced topological matters 1315], dynamics of topologically ordered systems [16 18, periodically driven closed quantum systems [19 25] and many body localization [26, 27]. (For review articles, we refer to 2833 .

Very recent experimental realisation of long-range interacting quantum models with tunable long-range interactions (or long-range pairing term) 34 has revived the interest in studying the non-equilibrium dynamics of quantum models with infinite-range interactions with interaction strength between two sites separated by a distance $r$ falling off in a power-law fashion as $1 / r^{\alpha}$ [35]47]. Historically, the study of a power-law interacting ferromagnetic Ising chain has a long histrory 48 51; Thouless 52] studied at length in the context of the possibility of long-range order in a one-dimensional system; it was argued that a finite critical temperature can not exist for $\alpha>2$. This was later confirmed through renormalisation group calculations [53. The most interesting case turns out to be the inverse-sqaure interacting Ising chain $(\alpha=2)$; this, on the one hand, mimics Kondo effect in metals [54, on the other, the transition from the ordered phase to the disordered phase is of KozterlitzThouless nature mediated by logarithmically interacting kinks and anti-kinks 55 60. The quantum phase transition (QPT) 61, 62 in the corresponding quantum version of the model has also been investigated where as well the situation $\alpha=2$, exhibits a unique behaviour 63 .

While a quantum Ising model with power-law interactions is non-integrable 35, 63, recently a generalised version of $p$-wave superconducting chain [64] of fermions with a long-range super-conducting pairing/gap term has been proposed [36 40]. The advantage of using this $p$-wave chain is the integrability and furthermore, the $2 \times 2$ structure corresponding to each momentum value in spite of the power-law interacting super-conducting term. What is noteworthy, that even though in the short-range limit $(\alpha \rightarrow \infty)$, the Kitaev chain can be mapped to the spin-1/2 XY chain through the Jordan-Wigner transformation, where the superconducting term represents the spin-spin interaction, this is not true for the long-range Kitaev chain; the latter, in fact, can not be mapped to a corresponding spin model.

In this work, we study the effect of long range interactions on the non-equilibrium dynamics of quantum models exploiting the integrability of the long-range $\mathrm{Ki}$ taev chain. In particular, we probe the Kibble-Zurek (KZ) 65 76] scaling of the defect density (or the residual energy) when the chain is linearly ramped across one of the quantum critical points (QCPs) and examine the dependence of $\mathrm{KZ}$ scaling exponent on the parameter $\alpha$. Furthermore, we subject the chain to a sudden quench across a QCP and analyse the dynamical quantum phase transitions (DQPTs) 77 82 (manifested in the non-analyticities in the logarithm of the rate function of return probability), in the sub-sequent temporal evolution with the final time independent Hamiltonian. We show that new features emerge as a consequence of the long-range interactions which disappear in the short-range limit. It is note-worthy, that the DQPTs [43, 45, 46] and Kibble-Zurek scaling [4] have been stud- 
ied for the long-range interacting quantum Ising chains and in both the situations, long-range interactions have been found to play a dominant role, at least for the smaller values of $\alpha$.

The paper is organised in the following fashion: in Sec. III we review the long-range interacting Kitaev chain and illustrate its quantum critical behaviour. Following a brief discussion on the KZ scaling, we analyze the same for the defect density generated following a slow ramping across the QCP in Sec. III] In Sec. IV] on the other hand, we shall consider a sudden quench and analyse the new features those emerge as a consequence of long-range interactions.

\section{LONG-RANGE KITAEV MODEL}

Let us first briefly review the long-range Kitaev chain of fermionic particles residing on a one-dimensional lattice [36 38]. Denoting fermionic annihilation(creation) operators as $c_{i}\left(c_{i}^{\dagger}\right)$, the Hamiltonian can be written as

$$
\begin{aligned}
H= & -J \sum_{i}\left(c_{i}^{\dagger} c_{i+1}+\text { h.c. }\right)-\mu \sum_{i}\left(n_{i}-\frac{1}{2}\right) \\
& +\frac{\Delta}{2} \sum_{i, l} d_{l}^{-\alpha}\left(c_{i} c_{i+l}+c_{i+l}^{\dagger} c_{i}^{\dagger}\right)
\end{aligned}
$$

where $n_{i}=c_{i}^{\dagger} c_{i}$ is the number operator for site $i$. Here, $J$ and $\mu$ denote the hopping strength of the fermionic particles between adjacent lattice sites and the onsite chemical potential, respectively, while $\Delta$ is the strength of the superconducting pairing term that decays with distance $l$ in a power law fashion characterized by exponent $\alpha$. In the limit of $\alpha \rightarrow \infty$, the model reduces to spinless $p$-wave superconductor Hamiltonian whose topological properties were unravelled by Kitaev [64]: In an open chain the model Hamiltonian has zero energy Majorana modes at the edges (and hence the chain is often referred to as a Majorana chain/wire). In this paper, we shall restrict our attention to a one-dimensional lattice with $L$ sites in a closed ring geometry (and hence topological properties of the model will not be exploited). The term $d_{l}$ in (1) measures the effective distance between two sites on the ring denoted by $i$ and $i+l$ and hence is given by $d_{l}=\min (l, L-l)$. Furthermore, we shall focus only on the case $\alpha>1$.

Even in the presence of the long-range interactions, the Hamiltonian (1) is quadratic in fermions and hence can exactly be solved by a Fourier Transformation and followed by Bogoliubov transformation in terms of fermionic operators in the momentum space $c_{i}=$ $\frac{1}{\sqrt{L}} \sum_{n=0}^{L-1} e^{-i k_{n} x_{i}} c_{k_{n}}$; we shall assume an anti-periodic boundary condition $\left(c_{i}=-c_{i+L}\right)$ so that the discrete momenta modes are quantized as $k_{n}=(2 \pi / L)\left(n+\frac{1}{2}\right)$. In the Fourier basis, the Hamiltonian (1) can be written in a block diagonal form,

$$
H=\frac{1}{2} \sum_{n=0}^{L-1} \Psi_{k_{n}}^{\dagger} H_{k_{n}} \Psi_{k_{n}}
$$

where $\Psi_{k_{n}}^{\dagger}=\left(\begin{array}{ll}c_{k_{n}}^{\dagger} & c_{-k_{n}}\end{array}\right)$ and $H_{k_{n}}$ is given by

$$
H_{k_{n}}=\left(\begin{array}{cc}
-\left(2 J \cos k_{n}+\mu\right) & i \Delta f_{\alpha}\left(k_{n}\right) \\
-i \Delta f_{\alpha}\left(k_{n}\right) & \left(2 J \cos k_{n}+\mu\right)
\end{array}\right),
$$

where $f_{\alpha}\left(k_{n}\right)=\sum_{l=1}^{L-1} \frac{\sin \left(k_{n} l\right)}{d_{l}^{\alpha}}$ is the Fourier transform of the superconducting gap term.

The Hamiltonian (2) for each block can be diagonalized by a Bogoliubov transformation

$$
\left(\begin{array}{c}
\eta_{k_{n}} \\
\eta_{-k_{n}}^{\dagger}
\end{array}\right)=\left(\begin{array}{cc}
\cos \theta_{k_{n}} & i \sin \theta_{k_{n}} \\
i \sin \theta_{k_{n}} & \cos \theta_{k_{n}}
\end{array}\right)\left(\begin{array}{c}
c_{k_{n}} \\
c_{-k_{n}}^{\dagger}
\end{array}\right)
$$

where $\tan \theta_{k_{n}}=-\frac{\Delta f_{\alpha}\left(k_{n}\right)}{2 J \cos k_{n}+\mu}$. The Hamiltonian in the final Bogoliubov basis is given by

$$
H=\sum_{n=0}^{L-1} \lambda_{\alpha}\left(k_{n}\right)\left(\eta_{k_{n}}^{\dagger} \eta_{k_{n}}-\frac{1}{2}\right)
$$

where $\lambda_{\alpha}\left(k_{n}\right)=\sqrt{\left(2 J \cos k_{n}+\mu\right)^{2}+\left(\Delta f_{\alpha}\left(k_{n}\right)\right)^{2}}$ are the eigen energy mode for each $\eta_{k_{n}}$ fermion. The ground state of the Hamiltonian is given by the vacuum of Bogoliubov $\eta_{k_{n}}$ fermions and is given by $|G S\rangle=$ $\prod_{n=0}^{L-1}\left(\cos \theta_{k_{n}}-i \sin \theta_{k_{n}} c_{k_{n}}^{\dagger} c_{-k_{n}}^{\dagger}\right)|0\rangle$ and $|0\rangle$ is vacuum of $c_{k_{n}}$ fermions. In the thermodynamic limit $L \rightarrow \infty$, when $k_{n}$ assume continuous values, the dispersion relation becomes

$$
\lambda_{\alpha}^{\infty}(k)=\sqrt{(2 J \cos k+\mu)^{2}+\left(\Delta f_{\alpha}^{\infty}(k)\right)^{2}}
$$

where $f_{\alpha}^{\infty}(k)=\lim _{L \rightarrow \infty} f_{\alpha}(k)=\frac{1}{i} \sum_{l=1}^{\infty} \frac{e^{i l k}-e^{-i l k}}{l^{\alpha}}=$ $-i\left(\mathbf{L i}_{\alpha}\left(e^{i k}\right)-\mathbf{L i}_{\alpha}\left(e^{-i k}\right)\right)$ with $\mathbf{L i}_{\alpha}(x)=\sum_{l=1}^{\infty} \frac{x^{l}}{l^{\alpha}}$ being the Polylogarithmic functions of $x$ that vanishes in the limit $k \rightarrow 0$ and $k \rightarrow \pi$. Focussing on the situation, $\alpha>1$, one finds that the spectrum is gapless for the parameter values $\mu=\mp 2 J$ for the modes $k=0$ and $\pi$, respectively, signalling the existence two quantum critical lines in $\mu-J$ plane. In the subsequent discussion, we shall concentrate on the QCP at $\mu=-2 J$ and analyze the spectrum close to the corresponding critical mode $k=0$. Let us reiterate that in the short-range limit $(\alpha \rightarrow \infty)$, the Hamiltonian exactly maps to spin $1 / 2$ transverse field XY model via Jordan-Wigner transformation. In this case, the critical lines separate the ferromagnetic $(|\mu|<2 J)$ and paramagnetic $(|\mu|>2 J)$ phases. What is interesting is that the location of the critical lines in the parameter space do not get altered even when $\alpha$ is finite. For brevity, we shall henceforth set $J=1$. 

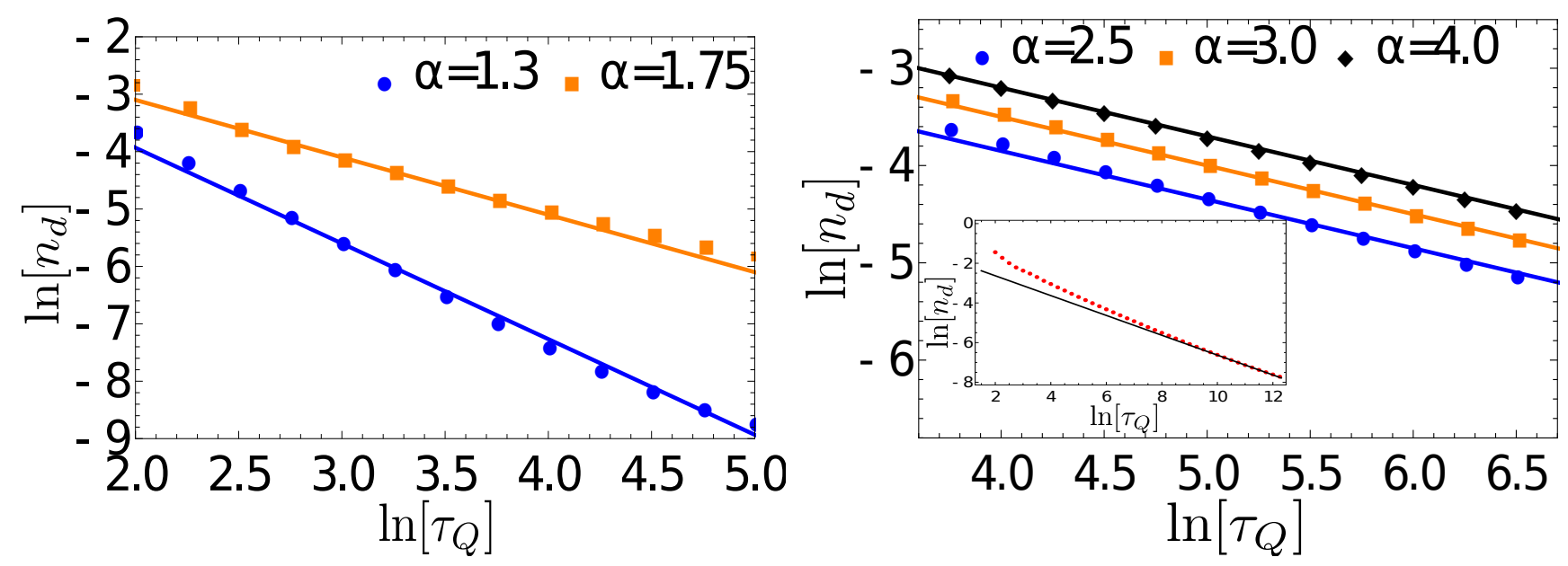

FIG. 1: The variation of the defect density $n_{d}$ with inverse quenching rate $\tau_{Q}$ : (Left) The figure shows that the scaling exponent (determined by the slope of the curve) depends on the interaction range $\alpha$ for $\alpha<2$. The solid lines show the theoretically predicted values $1 /(2 \alpha-2)$ and the dotted lines are from numerical solution of the differential Eqs. (9) using Runge-Kutta method with $L=10000, \mu_{i}=-10$ and $\mu_{f}=0$. (Right) The scaling exponent does no longer change with the range of interaction for $\alpha \geq 2$, rather gets saturated to the short range value $1 / 2$. In the marginal case $\alpha=2$, the exponent reaches the short-range limit of $1 / 2$ only in the asymptotic limit.

\section{RAMPING ACROSS THE CRITICAL POINT: KIBBLE-ZUREK SCALING}

When a quantum many-body system is quenched across a QCP by changing a parameter (say, linearly as $\left.t / \tau_{Q}\right)$, the dynamics is necessarily non-adiabatic due to the diverging relaxation time associated with the QCP; this results in the production of defect (or excess energy) in the final state of the system reached following the quench 65, 66. Remarkably, the density of defect exhibits a universal scaling with the inverse quenching rate $\tau_{Q}$ and some of the critical exponents associated with the QCP; this is known as the KZ scaling which has been studied extensively in recent years (For review, see 28 30].) (A quenching scheme is referred to as a slow ramping when the rate of quenching $\tau^{-1}$ is much slower than that associated with the minimum gap in the excitation spectrum of the Hamiltonian; however, close to the $\mathrm{QCP}$, the gap vanishes rendering the characteristic relaxation time infinite so that the dynamics is necessarily non-adiabatic however slow may the quenching be.)

To address the question, how does the long-range nature of interaction in $(1)$ modifies the $\mathrm{KZ}$ scaling relation, we employ a quenching protocol in which the chemical potential $\mu$ is tuned as $\mu(t)=-t / \tau_{Q}$, from a large negative value to a final value $\mu=0$; in the process the system, initially prepared in the ground state of the initial Hamiltonian, crosses the QCP at $\mu=-2$. As a result, the system gets excited in the vicinity of the critical point and the number of quasi-particles excited in the final state with $\mu=0$ is given by

$$
N=\sum_{k} \eta_{k}^{\dagger} \eta_{k}=\sum_{k} p_{k}
$$

where $p_{k}=\left\langle\psi_{f}\left|\eta_{k}^{\dagger} \eta_{k}\right| \psi_{f}\right\rangle$ is the excitation probability in the final state $\left|\psi_{f}\right\rangle$.

Referring to the quasi-momentum mode $k$, the state of the system at any instant $t$ can be written as $\left|\psi_{k}(t)\right\rangle=$ $u_{k}(t)|0\rangle+v_{k}(t)|1\rangle$, where $|0\rangle$ and $|1\rangle$ are the diabatic basis states $(1,0)^{T}$ and $(0,1)^{T}$. The corresponding Schrödinger equation is given by:

$$
\begin{aligned}
i \frac{d}{d t} u_{k}(t) & =-(2 J \cos k+\mu(t)) u_{k}(t)+i \Delta f_{\alpha}^{\infty}(k) v_{k}(t) \\
i \frac{d}{d t} v_{k}(t) & =-i \Delta f_{\alpha}^{\infty}(k) u_{k}(t)+(2 J \cos k+\mu(t)) v_{k}(t)
\end{aligned}
$$

with the initial condition $\left|u_{k}\right|^{2}=1$ and $\left|v_{k}\right|^{2}=0$. Using the transformation, $\tau=i \tau_{Q} \Delta f_{\alpha}^{\infty}(k)\left(t / \tau_{Q}-2 J \cos k\right)$, we can recast the above Eq. (8) to the standard LandauZener (LZ) form 83 85]

$$
\begin{aligned}
i \frac{d}{d \tau} u_{k}(t) & =-\left(\tau \tilde{\Delta}_{k}\right) u_{k}(t)+v_{k}(t) \\
i \frac{d}{d \tau} v_{k}(t) & =u_{k}(t)+\left(\tau \tilde{\Delta}_{k}\right) v_{k}(t)
\end{aligned}
$$

where $\tilde{\Delta}_{k}=\left(\tau_{Q}\left(\Delta f_{\alpha}^{\infty}(k)\right)^{2}\right)^{-1}$. For slow passage through the QCP, the excitation probability can be calculated using the LZ non-adiabatic transition probability [83, 84 of that the system ends in the excited state of the final Hamiltonian $\mu=0$;

$$
p_{k}=e^{-\pi / \tilde{\Delta}_{k}} \simeq e^{-\pi \tau_{Q}\left(\Delta f_{\alpha}^{\infty}(k)\right)^{2}} .
$$

In the large $\tau_{Q}$ limit, $p_{k}$ will be significant only for the modes close to the critical mode $k_{c}=0$ and hence we use the expansion formula of the polylogarithmic functions in 
the limit $k \rightarrow 0$, considering three limiting situations and calculate the corresponding scaling of the defect density: Situation I: $\alpha \notin \mathbb{Z}$ :

In this case, one can use the expansion:

$$
\begin{aligned}
\left(f_{\alpha}^{\infty}(k)\right)^{2}= & 4 \cos ^{2} \frac{\pi \alpha}{2} \Gamma^{2}(1-\alpha) k^{2(\alpha-1)}+4 \zeta^{2}(\alpha-1) k^{2} \\
& +8 \cos \frac{\pi \alpha}{2} \zeta(\alpha-1) \Gamma(1-\alpha) k^{\alpha}+o\left(k^{3}\right) \\
= & c_{1}(\alpha) k^{2(\alpha-1)}+c_{2}(\alpha) k^{\alpha}+c_{3}(\alpha) k^{2}+o\left(k^{3}\right)
\end{aligned}
$$

where we have used standard Gamma functions $(\Gamma)$ and Riemann zeta functions $(\zeta)$ [36, 86] and the coefficients are $c_{i}(\alpha)$ s given by:

$$
\begin{aligned}
& c_{1}(\alpha)=4 \cos ^{2} \frac{\pi \alpha}{2} \Gamma^{2}(1-\alpha), \\
& c_{2}(\alpha)=8 \cos \frac{\pi \alpha}{2} \zeta(\alpha-1) \Gamma(1-\alpha), \\
& c_{3}(\alpha)=4 \zeta^{2}(\alpha-1) .
\end{aligned}
$$

Using the expression of Eq.(11] in Eq. (7), the density of quasiparticle excitation in the final state at the end of the drive in the thermodynamic limit can be calculated as

$$
\begin{aligned}
n_{d}= & N / L=\frac{1}{2 \pi} \int_{-\pi}^{\pi} p_{k} d k \approx \int_{-\infty}^{\infty} p_{k} d k \\
\simeq & \frac{1}{2 \pi}\left(\frac{1}{\left(\pi c_{1}(\alpha) \tau_{Q}\right)^{\frac{1}{2 \alpha-2}}}+\frac{1}{\left(\pi c_{2}(\alpha) \tau_{Q}\right)^{\frac{1}{\alpha}}}+\right. \\
& \left.\frac{1}{\left(\pi c_{3}(\alpha) \tau_{Q}\right)^{\frac{1}{2}}}\right)
\end{aligned}
$$

where we have extended the range of integration over $k$ from $-\infty$ to $\infty$.

The expansion formula for polylogarithmic functions for integer $\alpha$ is given by

$$
\begin{aligned}
f_{\alpha}^{\infty}(k)= & -\frac{i^{\alpha} k^{\alpha-1}}{(\alpha-1) !}\left[\left(1+(-1)^{\alpha}\right)\left(\mathbb{H}_{\alpha-1}-\ln k-\frac{i \pi}{2}\right)+i \pi\right] \\
& -2 \sum_{\substack{m \in \text { odd } \\
m \neq \alpha-1}} \frac{\zeta(\alpha-m) i^{m+1}}{m !} k^{m}
\end{aligned}
$$

where $\mathbb{H}_{n}$ is nth Harmonic number. Using this expansion, we shall probe the following cases:

Situation II: $\alpha$ is an integer $\neq 2$ :

For any $\alpha \neq 2, \in \mathbb{Z}$ one finds:

$$
\left(f_{\alpha}^{\infty}(k)\right)^{2}=4(\zeta(\alpha-1))^{2} k^{2}+o\left(k^{3}\right) .
$$

Using Eq. (17), one can similarly calculate the scaling of the density of quasiparticle excitation in the final state

$$
n_{d} \simeq \frac{1}{\left(4 \pi(\zeta(\alpha-1))^{2} \tau_{Q}\right)^{\frac{1}{2}}}
$$

Situation III: Marginal case $\alpha=2$

The expansion of $\left(f_{\alpha}^{\infty}(k)\right)^{2}$ for $\alpha=2$ can be calculated from the expansion Eq. (16):

$$
\left(f_{2}^{\infty}(k)\right)^{2}=4(1-\ln k)^{2} k^{2}+o\left(k^{3}\right)
$$

We note that for $\alpha=2$, there is a prominent logarithmic correction in the leading order to the expression of $p_{k}$ which leads to sub-leading corrections to the scaling of $n_{d} \sim 1 / \sqrt{\tau_{Q}}$. However in the asymptotic limit of $\tau_{Q} \rightarrow \infty$, the sub-leading corrections drops off yielding the short-range scaling relation as shown in the inset of the right panel of Fig. 1 .

Let us now inspect the scaling relation predicted in Eqs. (15) and (18), recalling that in the $\alpha \rightarrow \infty$ limit $n_{d} \sim \tau^{-1 / 2}$; interestingly, we find that $1<\alpha<2$, the $\mathrm{KZ}$ scaling exponent is determined by the first term in Eq. (15) having the slowest decay with $\tau_{Q}$ in the limit of $\tau_{Q} \rightarrow \infty$. We thus have the $\mathrm{KZ}$ scaling exponent $1 /(2 \alpha-2)$, which reduces to the short-range value when $\alpha \rightarrow 2$.For $\alpha>2$, on the contrary, scaling exponent gets saturated to $1 / 2$. This establishes that the case $\alpha=2$ marks the boundary between the long-range and the short-range behavior so far as the KZ scaling is concerned. Furthermore, in the marginal case $\alpha=2$, there are non-universal sub-leading corrections which vanish in the limit of $\tau_{Q} \rightarrow \infty$. To verify the above mentioned analytically predicted scaling relations for different ranges of $\alpha$, we have numerically solved the differential Eqs. (9) to calculate the excitation probability and hence the defect density as shown in Fig. 11, we find that there is an excellent agreement between the numerical and analytical results.

\section{DYNAMICAL PHASE TRANSITION FOLLOWING A SUDDEN QUENCH}

Dynamical Quantum Phase transitions (DQPTs), introduced by Heyl et al. [77], in the context of non(16) analyticities in the temporal evolution of a quenched quantum system, is one of the emerging features of nonequilibrium dynamics of closed quantum systems. For a sudden quenching scheme, the system is prepared in the ground state $\left|\psi_{g}\right\rangle$ of the Hamiltonian $H_{i}$ corresponding to a parameter $\lambda=\lambda_{i}$. At time $t=0$, the parameter $\lambda$ of the Hamiltonian is suddenly changed from a value $\lambda_{i}$ to $\lambda_{f}$ while the state remains frozen at $\left|\psi_{f}\right\rangle=\left|\psi_{g}\right\rangle$. The initial state then has a non-trivial temporal evolution generated by the time-independent final Hamiltonian $H_{f}\left(\lambda_{f}\right)$ and one defines the so-called Loschmidt overlap amplitude(LOA) at an instant $t$ as $G(t)=\left\langle\psi_{f}\left|e^{-i H_{f} t}\right| \psi_{f}\right\rangle$. Generalising to the complex plane $G(z)=\left\langle\psi_{f}\left|e^{-H_{f} z}\right| \psi_{f}\right\rangle$, where $z=R+i t$ with $R$ being the real part, we can now define the dynamical free energy density in the thermodynamic limit for a 
$d$-dimensional system with linear dimension $L$

$$
f(z)=-\lim _{L \rightarrow \infty} \frac{1}{L^{d}} \ln G(z) .
$$

The zeros of $G(z)$ (which also indicate non-analyticities in $f(z)$ ) are so-called "Fisher zeros" (FZ) 87 89 which form a line in the complex $z$ plane for $d=1$ in the thermodynamic limit. In case, this line crosses the imaginary (real time) axis, which usually happens for quenches across a QCP, one observe cusp singularities in the rate function of the return probability defined as $I(t)=-\log |G(t)|^{2} / L$. Sharp non-analyticities of $I(t)$, at those instants of real time, referred to as DQPTs. Various aspects of DQPTs for several systems have been extensively studied in recent years [81, 82, 90, 110].

In this paper, we shall study the effect of long-range interactions, namely the parameter $\alpha$ on the DQPTs following a sudden quench of the parameter $\mu$ of the Hamiltonian from an initial value $\mu_{i}(<-2)$ to $\mu_{f}=1$ across a critical point $\mu=-2$ while the state of the system stays frozen in the ground state of the initial Hamiltonian. For the mode $k$, one can express the ground state for the mode $k,\left|\psi_{0}^{k}\right\rangle$ as,

$$
\left|\psi_{0}^{k}\right\rangle=u_{f}(k)\left|1_{k}^{f}\right\rangle+v_{f}(k)\left|2_{k}^{f}\right\rangle
$$

where $\left|1_{k}^{f}\right\rangle$ and $\left|2_{k}^{f}\right\rangle$ denote the ground(excited) state of the final Hamiltonian $H_{k}^{f}=H_{k}\left(\mu_{f}\right)$ with energy eigenvalues $\epsilon_{k}^{f}$ and $-\epsilon_{k}^{f}$, respectively, with $\left|u_{f}(k)\right|^{2}+\left|v_{f}(k)\right|^{2}=$ 1 ; the dynamical free energy in the complex $z$ plane is then given by

$$
f_{k}(z)=-\log \left(\left|v_{f}(k)\right|^{2} \exp \left(\epsilon_{k}^{f} z\right)+\left|u_{f}(k)\right|^{2} \exp \left(-\epsilon_{k}^{f} z\right)\right)
$$

Integrating over all the momenta modes we get,

$$
\begin{aligned}
& f(z)= \\
& \int_{0}^{\pi} \frac{d k}{2 \pi} \log \left(\left|v_{f}(k)\right|^{2} \exp \left(\epsilon_{k}^{f} z\right)+\left|u_{f}(k)\right|^{2} \exp \left(-\epsilon_{k}^{f} z\right) .\right) \\
= & -\int_{0}^{\pi} \frac{d k}{2 \pi} \log \left(\left(1-p_{k}\right) \exp \left(\epsilon_{k}^{f} z\right)+p_{k} \exp \left(-\epsilon_{k}^{f} z\right)\right) ;
\end{aligned}
$$

The zeros of the dynamical partition function $G(z)$ (i.e., FZs) is then given by

$$
z_{n}(k)=\frac{1}{2 \epsilon_{k}^{f}}\left[\log \left(\frac{p_{k}}{1-p_{k}}\right)+i \pi(2 n+1)\right]
$$

where $n=0,1,2 \ldots, p_{k}=\left|v_{f}(k)\right|^{2}$ is the probability of excitation. The critical mode $k=0$ is frozen and $p_{k}=1$ while far away from critical modes $p_{k} \rightarrow 0$. This implies that as the lattice momentum varies from 0 to $\pi$, the real values of FZs given in Eq. 23 span from $-\infty$ to $\infty$ along the real time axis. For an intermediate value $k=k_{*}$ when $p_{k_{*}}=1 / 2$, the lines of FZs lie right on the imaginary (real) time axis; the corresponding instants of real time are

$$
t_{n}^{*}=\frac{\pi}{2 \epsilon_{k_{*}}^{f}}(2 n+1) .
$$

The rate function of the return probability in this case can be evaluated exactly in the form 77

$$
\begin{aligned}
I(t) & =-\frac{\log |G(t)|^{2}}{L}=2 \operatorname{Re} f(z) \\
& =-\int_{0}^{\pi} \frac{d k}{2 \pi} \log \left(1+4 p_{k}\left(p_{k}-1\right) \sin ^{2}\left(\epsilon_{k}^{f} t\right)\right)
\end{aligned}
$$

are non-analytic due to non-analytic contribution for the mode $k_{*}$ at the real instants $t_{n}^{*}$ described by Eq. (24). The physical significance of the mode $k_{*}$ with $p_{k_{*}}=1 / 2$ is that both the ground and the excited states of the final Hamiltonian for the mode $k_{*}$ are equally populated; (this mode $k_{*}$, in that sense, is at infinite temperature with respect to the final Hamiltonian). The existence of a momentum mode $k_{*}$ ensures the existence of DQPTs; if the quenching amplitude is such that there exists no $k_{*}$, the DQPTs will be absent in the subsequent evolution generated by the final Hamiltonian.

From the $2 \times 2$ Hamiltonian 2 we find that the ground state and excited states of the initial and final Hamiltonian, $\left|1^{i / f}\right\rangle$ and $\left|2^{i / f}\right\rangle$, respectively, can be decomposed to the form using the diabatic basis vector $(1,0)^{T}$ and $(0,1)^{T}$ (where $T$ denotes the transpose) in the form:

$$
\begin{aligned}
\left|1^{i / f}\right\rangle & =\cos \frac{\theta_{k}^{i / f}}{2}(1,0)^{T}-\sin \frac{\theta_{k}^{i / f}}{2}(0,1)^{T} \\
\left|2^{i / f}\right\rangle & =\sin \frac{\theta_{k}^{i / f}}{2}(1,0)^{T}+\cos \frac{\theta_{k}^{i / f}}{2}(0,1)^{T}
\end{aligned}
$$

where $\tan \theta_{k}=\Delta_{k} /(\mu+2 \cos k)$ and $\Delta_{k}=\Delta f_{\alpha}(k)$ depends on $\alpha$. The excitation probability $p_{k}$ in this notation simplified as,

$$
\begin{aligned}
& p_{k}=\left|\left\langle 1^{i} \mid 2^{f}\right\rangle\right|^{2}=\sin ^{2}\left[\left(\theta_{k}^{i}-\theta_{k}^{f}\right) / 2\right] \\
= & \frac{1}{2}\left[1-\frac{\left(\mu_{i}+2 \cos k\right)\left(\mu_{f}+2 \cos k\right)+\Delta_{k}^{2}}{\sqrt{\left(\left(\mu_{i}+2 \cos k\right)^{2}+\Delta_{k}^{2}\right)\left(\left(\mu_{f}+2 \cos k\right)^{2}+\Delta_{k}^{2}\right)}}\right]
\end{aligned}
$$

From the above discussion, one can conclude the condition for DQPT as there exists definite momentum mode(s) defined as $k=k_{*}$ for which the $p_{k_{*}}=1 / 2$ and for this mode we have the condition $\left(\mu_{i}+2 \cos k_{*}\right)\left(\mu_{f}+\right.$ $\left.2 \cos k_{*}\right)+\Delta_{k_{*}}^{2}=0$ for all values of $\alpha>1$. We note that for $\alpha \rightarrow \infty$, the prominent occurrence of DQPTs have already been established [77, 96].

However, there is an interesting possibility that arises due to the long-range nature of the model; we illustrate this below assuming for simplicity $\mu_{f}=1$. Let us refer 

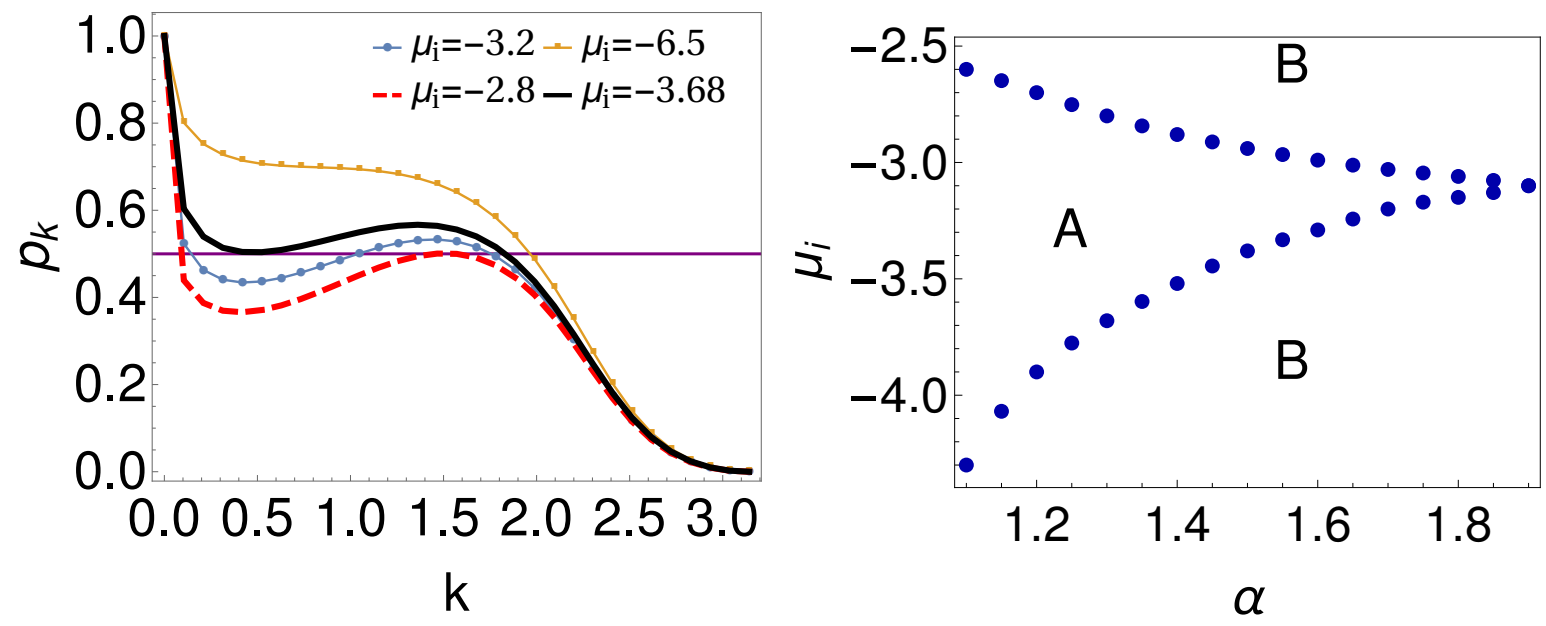

FIG. 2: (left) Plot of the transition probability 27) as a function of $k$ for different values of $\mu_{i}$ with $\mu_{f}=1$; the long-range interaction parameter $\alpha$ is fixed to $\alpha=1.3$. We find two distinct regimes: (i) The transition probability $p_{k}$ crosses the value $1 / 2$ for three values of $k$ (i.e., yielding three values of $k_{*}$ ) for $\mu_{i}=-3.2$ and once for $\mu_{i}=-6.5$, while for other two values it crosses the $p_{k}=1 / 2$ once and touches at another value of $k$. (Right) Phase boundary separating the three crossings region (A) from the single crossing regions (B) is shown in the $\alpha-\mu_{i}$ plane. On the phase boundary $p_{k}$ crosses (and touches) the $p_{k}=1 / 2$ line twice as shown in the left panel. It is noteworthy that the width of region A shrinks as $\alpha$ increase and vanishes as $\alpha \rightarrow 2$.

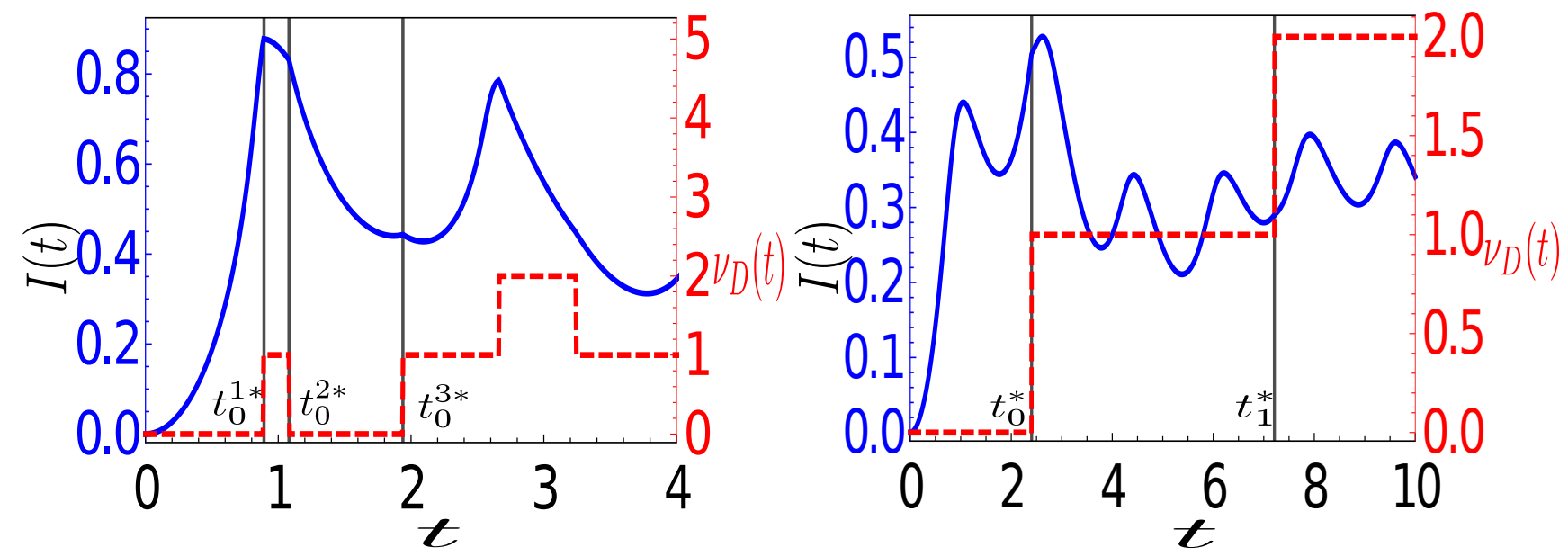

FIG. 3: (left) The temporal evolution of $\nu_{D}(t)$ as a function of $t$ corresponding to the region A shown in the right panel of Fig. 2 We find that corresponding to $n=0$, there are three instants $t_{0}^{1 *}, t_{0}^{2 *}$ and $t_{0}^{3 *}$ at which $\nu_{D}(t)$ makes discontinuous changes by a factor of unity. However, whether the DTOP jumps or drops is determined by the slope of the $p_{k}$ vs $k$ curve at $k_{*}$ (i.e., the crossing points) as depicted in the left panel of the Fig. 2. (right) $\nu_{D}$ vs $t$ for the region B the right panel of Fig. 2 . we have only one instant of non-analyticity corresponding to each $n$ and we show jumps in the DTOP corresponding to $n=0$ and $n=1$; notably in this case there is no drop in $\nu_{D}(\mathrm{t})$ at DQPTs.

to the left panel of Fig. 2 interestingly, we find that there exists a region in the parameter space of $\mu_{i}$, where there are three values of $k_{*}$ (as $k$ ranges from 0 to $\pi$ ) for which $p_{k_{*}}=1 / 2$ even though the system is quenched across a single QCP. For each of the values of $k_{*}$, we have different values of $t_{n}^{*}$ for the same value of $n$ as obtained from Eq. (24). Usually, one encounters a single instant of non-analyticity for a given $n$, i.e., there is a single $k_{*}$ as $k$ ranges from 0 to $\pi$, following a quench across a QCP.

In the right panel of Fig. 2, we plot a phase diagram in $\mu_{i}-\alpha$ plane with $\mu_{f}=1$, separating these two regions: (i) in region $\mathrm{A}$, there are three $k_{*}$ values leading to three instants of real time at which DQPTs occur for a given $n$ as shown in the left panel of Fig. 3. (ii) On the contrary in region $\mathrm{B}$, there is only $k_{*}$ as in the short-range case (right panel Fig. 3). Finally, on the phase boundary 
separating these two regions, there are two values of $k_{*}$ with $p_{k_{*}}=1 / 2$. What is note worthy that $\alpha \rightarrow 2$, the width of the region $\mathrm{A}$ shrinks and vanishes confirming that the occurrence of a region A with three DQPTs for a given $n$ is indeed an artefact of long-range nature of the Hamiltonian.

Let us finally address the question whether the DQPTs occurring in this model can be characterised by the dynamical topological order parameter (DTOP) introduced in Ref. 100, and how does its behaviour change in the region $\mathrm{A}$ as compared to the region $\mathrm{B}$. We would like to mention that the DTOP can be interpreted as a non-local order parameter and its temporal variation appropriately characterises every instant at which DQPTs occur. More precisely, it stays fixed to a quantised integer value between two instants of successive DQPTs while at every DQPT it shows a discontinuous jump of unit magnitude. Writing the LOA for each $k$ mode in the polar form as $f_{k}(t)=\left|r_{k}(t)\right| \exp i \phi_{k}(t)$ where $\phi_{k}(t)$ is given by the formula

$$
\phi_{k}(t)=\tan ^{-1}\left\{\left(\left|v_{f}(k)\right|^{2}-\left|u_{f}(k)\right|^{2}\right) \tan \left(\epsilon_{k}^{f} t\right)\right\},
$$

we can define the gauge-independent Pancharathnam geometric phase (PGP) in the form $\phi_{k}^{G}(t)=\phi_{k}(t)-$ $\phi_{k}^{\text {dyn }}(t)$, where the dynamical phase $\phi_{k}^{\text {dyn }}(t)=$ $-\int_{0}^{t} d s\left\langle\psi_{f}^{k}(s)\left|H_{k}^{f}\right| \psi_{f}^{k}(s)\right\rangle=\left(\left|v_{f}(k)\right|^{2}-\left|u_{f}(k)\right|^{2}\right) \epsilon_{k}^{f} t$. The DTOP $\left(\nu_{D}(t)\right)$ is then defined as the winding number

$$
\nu_{D}(t)=\frac{1}{2 \pi} \int_{0}^{\pi} \frac{\partial \phi_{k}^{G}}{\partial k}
$$

The phase $\phi_{k}^{G}$ is pinned to zero for the momentum values $k=0$ and $\pi$,i.e., $\nu_{D}(t)$ defined in Eq. 29) stays constant as long as the system does hit a non-analyticity at the critical time when there is a discontinuous jump [100, 103] as shown in Fig. 3, whether the jump will be increasing or decreasing $(+1$ or -1$)$ will be determined by the slope of the $p_{k}$, i.e., $\partial p_{k} / \partial k$ at the corresponding critical momentum $k_{*}$. Referring to the left panel of Fig. 3, the first three non-analyticities in $I(t)$ correspond to the sector $n=0$ as obtained from Eq. 24) for three values of $k_{*}$ as depicted in the curve corresponding to $\mu_{i}=-3.2$ in the left panel of the Fig. 2. Labelling these instances, derived from (24), as $t_{0}^{1 *}, t_{0}^{2 *}$ and $t_{0}^{3 *}$, respectively, we observe that the DTOP jumps at $t_{0}^{1 *}$ and $t_{0}^{3 *}$ while at $t_{0}^{2 *}$ it drops, in all the cases by a factor of unity. The behaviour of the DTOP, i.e, whether $\nu_{D}$ would jump or drop, is determined by the slope the curve $p_{k}$ vs $k$ at $k_{*}$ [100, 103]; following the same curve in Fig. 2, we find the slope is different for the middle crossing in comparison to the other two crossings which results in the change of polarity of the jumps in $\nu_{D}(t)$ at a DQPTs. On the contrary, for region $\mathrm{B}$, we have only crossing for one value of $n$ and we observe successive jumps of the same polarity at every DQPTs as shown in the right panel of Fig. 3

\section{CONCLUDING COMMENTS}

Using the integrability and the $2 \times 2$ nature of a Kitaev chain with a long-range super-conducting term, we have studied the effect of long-range interactions on the $\mathrm{KZ}$ scaling for slow quenching and DQPTs following a sudden quenching. In both the situations, we have established that the long-range interactions characterised by the parameter $\alpha$ plays a non-trivial role. In the longrange interacting case $1<\alpha<2$, we show that the $\mathrm{KZ}$ scaling exponent dictating the power-law decay of the defect density $\left(n_{d}\right)$ with the inverse rate of quenching $\left(\tau_{Q}\right)$ depends non-trivially on $\alpha$ and is given by $1 /(2 \alpha-2)$. In the limit of $\alpha \geq 2$, i.e., in the short-range limit, the exponent saturates to the short-range value $1 / 2$. Therefore, the value $\alpha=2$ marks the boundary between the long-range and the short-range behaviour of the scaling of $n_{d}$. It is note-worthy that the scaling of $n_{d}$ in the marginal case $\alpha=2$ has non-universal sub-leading corrections which vanish in the limit $\tau_{Q} \rightarrow \infty$. Let us recall that the importance of the case $\alpha=2$ for a classical one dimensional Ising model emerges from a renormalisation group calculations (and the transition at $\alpha=2$ is of Kosterlitz Thouless nature). However, the present work deals with a quantum model in a non-equilibrium situation where the importance of the $\alpha=2$ scenario emerges from the fact that the scaling behavior of the excitation spectrum at the quantum critical point (with the momentum) changes precisely at $\alpha=2$. Consequently the case $\alpha=2$ points to a crossover from the long-range to the short-range behavior. Whether this can be put in a generic renormalization scenario for a non-equilibrium situation is indeed a pertinent question for future research.

For DQPTs, we observe an interesting three crossing region where there exist three instants of nonanalyticities in $I(t)$ for a given $n$. This is a consequence of three possible values of $k_{*}$ in the $p_{k}-k$ plane; as a result, we find a region with 3-DQPT for a given $n$ in the $\left(\mu_{i}-\alpha\right)$ plane (with fixed $\left.\mu_{f}\right)$ which are also detected by the DTOP. Interestingly, the width of this region shrinks with increasing $\alpha$ and vanishes in as $\alpha \rightarrow 2$ confirming that this unusual region emerges as a result of the long-range interactions. Given the recent experimental realisation of the long-range interacting systems 34] and experimental detection of DQPTs [111, 112, we believe that our studies can be experimentally verified.

\section{Acknowledgments}

We acknowledge Utso Bhattacharya for helpful comments and discussions and SERB, DST, New Delhi for financial support. 
[1] I. Bloch, J. Dalibard, and W. Zwerger, Rev. Mod. Phys. 80, 885 (2008).

[2] M. Lewenstein, A. Sanpera, and V. Ahufinger, (Oxford University Press, Oxford (2012)).

[3] M. Greiner , O. Mandel, T. W. Hansch, and I. Bloch, Nature 419, 51 (2002).

[4] T. Kinoshita, T. Wenger, and D. S. Weiss, Nature 440, 900 (2006).

[5] M. Gring, M. Kuhnert, T. Langen, T. Kitagawa, B. Rauer, M. Schreitl, I. Mazets1, D. Adu Smith, E. Demler, and J. Schmiedmayer, Science 337, 1318 (2012).

[6] S. Trotzky, Y-A. Chen, A. Flesch, I. P. McCulloch, U. Schollwck, J. Eisert, and I. Bloch, Nature 8, 325 (2012).

[7] M. Cheneau, P. Barmettler, D. Poletti, M. Endres, P. Schauss, T. Fukuhara, C. Gross, I. Bloch, C. Kollath, and S. Kuhr, Nature 481, 484 (2012).

[8] M. Schreiber, S. S. Hodgman, P. Bordia, Henrik P. Lüschen, M. H. Fischer, R. Vosk, E. Altman, U. Schneider, I. Bloch, Science 349, 842 (2015).

[9] D. Fausti, R. I. Tobey, , N. Dean, S. Kaiser, A. Dienst, M. C. Hoffmann, S. Pyon, T. Takayama, H. Takagi,, A. Cavalleri, Science 331, 189 (2011).

[10] M. C. Rechtsman, J. M. Zeuner, Y. Plotnik, Y. Lumer, D.Podolsky, F. Dreisow, S. Nolte, M. Segev, and A. Szameit, Nature 496196 (2013).

[11] P. Calabrese and J. Cardy, Phys. Rev. Lett. 96, 136801 (2006); J. Stat. Mech, P06008 (2007).

[12] M. Rigol, V. Dunjko, and M. Olshanii, Nature 452, 854 (2008).

[13] T Oka and H Aoki, Phys. Rev. B 79, 081406 (2009).

[14] T. Kitagawa, E. Berg, M. Rudner, and E. Demler, Phys. Rev. B 82, 235114 (2010).

[15] N. H. Lindner, G. Refael, and V. Galitski, Nat. Phys. 7, 490-495, (2011).

[16] A. Bermudez, D. Patane, L. Amico, and M. A. MartinDelgado, Phys. Rev. Lett. 102, 135702, (2009).

[17] A. A. Patel, S. Sharma, and A. Dutta, Eur. Phys. Jour. B 86, 367 (2013); A. Rajak and A. Dutta, Phys. Rev. E 89, 042125 (2014); P. D. Sacramento, Phys. Rev. E 90 032138, (2014); M. D. Caio, N. R. Cooper, and M. J. Bhaseen, Phys. Rev. Lett. 115, 236403 (2015).

[18] M. Thakurathi, A. A. Patel, D. Sen, and A. Dutta, Phys. Rev. B 88, 155133 (2013).

[19] V. Mukherjee and A. Dutta, J. Stat. Mech. P05005 (2009).

[20] A. Das, Phys. Rev. B 82, 172402 (2010).

[21] A. Russomanno, A. Silva, and G. E. Santoro , Phys. Rev. Lett. 109, 257201 (2012).

[22] S. Sharma, A. Russomanno, G. E. Santoro, and A. Dutta, EPL 106, 67003 (2014).

[23] M. Bukov, L. D'Alessio, and A. Polkovnikov, Adv. Phys. 64 , No. 2, 139-226 (2016).

[24] A. Dutta, A. Das, and K. Sengupta, Phys. Rev. E,110, 012104 (2015).

[25] A. Sen, S. Nandy, and K. Sengupta, Phys. Rev. B,94, 214301 (2016).

[26] A. Pal and D. A. Huse, Phys. Rev. B 82, 174411 (2010).

[27] R. Nandkishore and D. A. Huse, Annual Review of Condensed Matter Physics, 6, 15-38 (2015).

[28] J. Dziarmaga, Advances in Physics 59, 1063 (2010).

[29] A. Polkovnikov, K. Sengupta, A. Silva, and M. Ven- galattore, Colloquium: Nonequilibrium dynamics of closed interacting quantum systems, Rev. Mod. Phys. 83, 863 (2011).

[30] A. Dutta, G. Aeppli, B. K. Chakrabarti, U. Divakaran, T. Rosenbaum, and D. Sen, Quantum Phase Transitions in Transverse Field Spin Models: From Statistical Physics to Quantum Information (Cambridge University Press, Cambridge, 2015).

[31] J. Eisert, M. Friesdorf, and C. Gogolin, Nat. Phys. 11, 124 (2015).

[32] L. D'Alessio, Y. Kafri, A. Polkovnikov, and M. Rigol, Adv. Phys. 65, 239 (2016).

[33] Quantum Integrability in Out of Equilibrium Systems Edited by P. Calabrese, F. H. L. Essler, and G. Mussardo, Special issue of J. Stat. Mech. Th. and Exp. 2016, (2016).

[34] P. Richerme, Z.-X. Gong, A. Lee, C. Senko, J. Smith, M. Foss-Feig, S. Michalakis, A. V. Gorshkov, and C. Monroe, Nature 511, 198 (2014).

[35] B Žunkovič, A. Silva, and M. Fabrizio, Phil. Trans. R. Soc. A 374, 20150160 (2016).

[36] D. Vodola, L. Lepori, E. Ercolessi, and G. Pupillo, New J. Phys. 18, 015001 (2016).

[37] O. Viyuela, D. Vodola, G. Pupillo, and M. A. MartinDelgado, Phys. Rev. B 94, 125121 (2016).

[38] L. Lepori, A. Trombettoni, and D. Vodola, J. Stat. Mech. 033102 (2017).

[39] M. V. Regemortel, M. Wouters, and D. Sels,Phys. Rev. A 93, 032311 (2016).

[40] S. Nandy, A. Sen, and K. Sengupta, unpublished,(2017).

[41] Antonio Alecce, Luca Dell'Anna, arXiv:1703.10086 (2017).

[42] S. Fey and K. P. Schmidt, Phys. Rev. B 94, 075156 (2016)

[43] B Žunkovič, M. Heyl, M. Knap, A. Silva, arXiv:1609.08482 (2016)

[44] J. C. Halimeh, V. Zauner-Stauber, I. P. McCulloch, I. de Vega, U. Schollwck, and M. Kastner, Phys. Rev. B 95, 024302 (2017).

[45] J. C. Halimeh and V. Zauner-Stauber, arXiv:1610.02019 (2017).

[46] I. Homrighausen, N. O. Abeling, V. Zauner-Stauber, and J. C. Halimeh, arXiv:1703.0919 (2017).

[47] D. Jaschke, K. Maeda, J. D. Whalen, M. L. Wall, and L. D. Carr, New J. Phys. 19 , 033032 (2017).

[48] D. Ruelle, Commun. Math. Phys. 9, 267 (1968).

[49] F. J. Dyson, Commun. math. Phys. 12, 91 ?107 (1969).

[50] F. J. Dyson, Commun. math. Phys. 12, 212-215 (1969).

[51] M. Kac, and C. J. Thompsom, J. Math. Phys. 10, 8 (1969).

[52] D. J. Thouless, Phys. Rev. 187, 732 (1969).

[53] M.E. Fisher, S.K. Ma, and B.G. Nickel, Phys. Rev. Lett. 29, 917 (1972).

[54] P.W. Anderson and G. Yuval, J. Phys. C 4, 607 (1971).

[55] J.M. Kosterlitz, Phys. Rev. Lett. 37, 1577 (1976).

[56] J.L. Cardy, J. Phys. A 14, 1407 (1981).

[57] J. Bhattacharjee, S. Chakravarty, J.L. Richardson, and D.J. Scalapino, Phys. Rev. B 24, 3862 (1981).

[58] J.K. Bhattacharjee, J.L. Cardy, and D.J. Scalapino, Phys. Rev. B 25, 1681 (1982)

[59] J.Z. Imbrie and C.M. Newmann, Commun. Math. Phys. 
118, 303 (1988).

[60] E. Luijten and H. Meingfeld, Phys. Rev. Lett. 86, 5305 (2001).

[61] S. Sachdev, Quantum Phase Transitions (Cambridge University Press, Cambridge, UK, 2011).

[62] S. Suzuki, J-i Inoue, and Bikas K. Chkarabarti, Quantum Ising Phases and Transitions in Transverse Ising Models (Springer, Lecture Notes in Physics, Vol. 862 (2013)).

[63] A. Dutta and J. K. Bhattacharjee, Phys. Rev. B 64, 184106 (2001).

[64] A. Kiteav, arXiv:cond-mat/0010440 (2000); A. Kitaev, C. Laumann, arXiv:0904.2771, Les Houches Summer School "Exact methods in low-dimensional physics and quantum computing" , 2008.

[65] T. W. B. Kibble, J. Phys. A: Math. Gen. 9, 1387 (1976); Phys. Rep. 67, 183 (1980).

[66] W. H. Zurek, Nature (London) 317, 505 (1985); Acta Phys. Pol. B 24, 1301 (1993); Phys. Rep. 276, 177 (1996).

[67] B. Damski, Phys. Rev. Lett. 95, 035701 (2005).

[68] W.H. Zurek, Phys. Rev. Lett. 95, 105701 (2005).

[69] W. H. Zurek, U. Dorner, and P. Zoller, Phys. Rev. Lett. 95, 105701 (2005).

[70] A. Polkovnikov, Phys. Rev. B 72, 161201(R) (2005).

[71] B. Damski and W. H. Zurek, Phys. Rev. A 73, 063405 (2005).

[72] V. Mukherjee, U. Divakaran, A. Dutta, and D. Sen Phys. Rev. B 76, 174303 (2007).

[73] U. Divakaran, A. Dutta, and D. Sen Phys. Rev. B 78, 144301 (2008).

[74] K. Sengupta, D. Sen, and S. Mondal Phys. Rev. Lett. 100, 077204 (2008).

[75] D. Sen, K. Sengupta, and S. Mondal Phys. Rev. Lett. 101, 016806 (2008).

[76] A. Dutta,A. Rahmani, and A. del Campo Phys. Rev. Lett. 117, 080402 (2016).

[77] M. Heyl, A. Polkovnikov, and S. Kehrein, Phys. Rev. Lett., 110, 135704 (2013).

[78] C. Karrasch and D. Schuricht, Phys. Rev. B, 87, 195104 (2013).

[79] N. Kriel, C. Karrasch, and S. Kehrein, Phys. Rev. B 90, 125106 (2014).

[80] M. Heyl, Phys. Rev. Lett., 113, 205701 (2014).

[81] F. Pollmann, S. Mukerjee, A. G. Green, and J. E. Moore, Phys. Rev. E 81, 020101(R) (2010).

[82] U. Divakaran, S. Sharma, and A. Dutta, Phys. Rev. E 93, 052133 (2016).

[83] C. Zener, Proc. Roy. Soc. London Ser A 137, 696 (1932); L. D. Landau and E. M. Lifshitz, Quantum Mechanics: Non-relativistic Theory, 2nd ed. (Pergamon Press, Oxford, 1965).

[84] S. Suzuki and M. Okada, in Quantum Annealing and Related Optimization Methods, Ed. by A. Das and B. K. Chakrabarti (Springer-Verlag, Berlin, 2005), p. 185.
[85] N. V. Vitanov and B. M. Garraway, Phys. Rev. A 53, 4288 (1996); N. V. Vitanov, ibid. 59, 988 (1999).

[86] F. W. J. Olver, D. W. Lozier, R. F. Boisvert, and C. W. Clark, NIST Handbook of Mathematical Functions (Cambridge University Press, Cambridge, England, 2010); M. Abramowitz and I. A. Stegun, Handbook of Mathematical Functions(Dover, 1964).

[87] M.E. Fisher, in Boulder Lectures in Theoretical Physics (University of Colorado, Boulder, 1965), Vol. 7.

[88] C. Yang and T. Lee, Phys. Rev. 87, 404 (1952).

[89] W. van Saarloos and D. Kurtze, J. Phys. A 17, 1301 (1984).

[90] M. Heyl, Phys. Rev. Lett., 115, 140602 (2015) .

[91] T. Palmai, Phys. Rev. B 92, 235433 (2015).

[92] Z. Huang and A. V. Balatsky, Phys. Rev. Lett. 117, 086802 (2016).

[93] T. Puskarov and D. Schuricht, SciPost Phys. 1, 003 (2016).

[94] J. M. Zhang and H.-T. Yang, EPL 116, 10008 (2016).

[95] M. Heyl, Phys. Rev. B 95, 060504 (2017).

[96] S. Vajna, and B. Dora, Phys. Rev. B 89, 161105(R) (2014).

[97] S. Sharma, S. Suzuki, and A. Dutta, Phys. Rev. B 92, 104306 (2015).

[98] S. Vajna and B. Dora, Phys. Rev. B 91, 155127 (2015).

[99] M. Schmitt and S. Kehrein, Phys. Rev. B 92, 075114 (2015).

[100] J. C. Budich and M. Heyl, Phys. Rev. B 93, 085416 (2016).

[101] F. Andraschko and J. Sirker, Phys. Rev. B 89, 125120 (2014).

[102] E. Canovi, P. Werner, and M. Eckstein, Phys. Rev. Lett. 113, 265702 (2014).

[103] S. Sharma, U. Divakaran, A. Polkovnikov, and A. Dutta, Phys. Rev. B 93, 144306 (2016).

[104] A.A. Zvyagin, Low Temp. Phys. 42, 971-994 (2016).

[105] T. Obuchi, S. Suzuki, K. Takahashi, arXiv:1702.05396 (2017).

[106] Thoms Fogarty, Ayaka Usui, Thomas Busch, Alessandro Silva, John Goold, arXiv:1704.07659 (2017).

[107] U. Bhattacharya and A. Dutta, arXiv:1610.02674 (2016).

[108] U. Bhattacharya and A. Dutta, arXiv:1701.03911 (2017).

[109] U. Bhattacharya, S. Bandyopadhyay and A. Dutta, arXiv:1705.04555 (2017).

[110] M. Heyl and J. C. Budich, arXiv:1705.08980 (2017).

[111] P. Jurcevic, H. Shen, P. Hauke, C. Maier, T. Brydges, C. Hempel, B. P. Lanyon, M. Heyl, R. Blatt, C. F. Roos, arXiv:1612.06902 (2016).

[112] N. Fläschner, D. Vogel, M. Tarnowski, B, S. Rem, D.-S. Lühmann, M. Heyl, J. Budich, L. Mathey, K. Sengstock, C. Weitenberg, arXiv:1608.05616 (2016). 